\title{
A New Perspective on Neonatal Smiling: Differences Between the Judgments of Expert Coders and Naive Observers
}

\author{
Marco Dondi \\ Dipartimento di Scienze Umane, Università di Ferrara \\ Daniel Messinger \\ Department of Psychology and Pediatrics, University of Miami \\ Marta Colle, Alessia Tabasso, and Francesca Simion \\ Dipartimento di Psicologia dello Sviluppo e della Socializzazione, \\ Università di Padova \\ Beatrice Dalla Barba \\ Dipartimento di Pediatria, Università di Padova \\ Alan Fogel \\ Department of Psychology, University of Utah
}

\begin{abstract}
To better understand the form and recognizability of neonatal smiling, 32 newborns (14 girls; $M=25.6 \mathrm{hr}$ ) were videorecorded in the behavioral states of alertness, drowsiness, active sleep, and quiet sleep. Baby Facial Action Coding System coding of both lip corner raising (simple or non-Duchenne) and lip corner raising with cheek raising (Duchenne smile) was followed by a smile recognition task using 48 naive observers. Both types of smiles were detected in all behavioral states. Lip corner raising with cheek raising (Duchenne smiling) tended to predominate in active (rapid eye movement) sleep, suggesting a potential tie to early constituents of emotion. A significant portion of the typically briefer lip corner raising distinguished by expert coders was not recognized as smiling by the naive observers. These briefer actions may represent a motor phenomenon idiosyncratic to the neonatal period.
\end{abstract}

Send correspondence to Marco Dondi, Dipartimento di Scienze Umane, Università di Ferrara, via Savonarola 38, 44100 Ferrara, Italy. E-mail: marco.dondi@unife.it 
Smiling is a stable pattern of facial movements present at birth, in term (Emde \& Koenig, 1969a, 1969b; Korner, 1969; Wolff, 1963, 1966) and preterm neonates (Emde, McCartney, \& Harmon, 1971; Wolff, 1987). Unlike social smiling, which emerges later (at about 2 months of age) and has been analyzed in several early (Ambrose, 1961; Gewirtz, 1965) and more recent empirical contributions (Fogel, Nelson-Goens, Hsu, \& Shapiro, 2000; Fox \& Davidson, 1988; Messinger, Fogel, \& Dickson, 1999, 2001; Sroufe \& Waters, 1976), little is known about the significance, form, and recognizability of neonatal smiles or their developmental relevance for later smiling.

Behavioral states are crucial organizers of infant behavior (Brazelton, 1983; Prechtl \& Beintema, 1964; Wolff, 1987). Like other early spontaneous motor patterns exhibited during neonatal sleep, such as startles, mouthing, and reflexive sucks, neonatal smiling is thought to occur in the absence of recognized external or internal (visceral) stimuli (Korner, 1969; Wolff, 1963, 1966). For this reason, neonatal smiling is known as reflexive, spontaneous, or endogenous smiling (Emde \& Harmon, 1972; Fogel \& Thelen, 1987; Sroufe, 1979, 1996; Wolff, 1987). Observational evidence suggests that newborn smiling is associated with the behavioral state of active sleep (irregular, paradoxical, or rapid eye movement [REM] sleep) and drowsiness. Early research indicated, in fact, that neonatal smiling occurred almost exclusively during REM states (Emde \& Koenig, 1969a, 1969b; Korner, 1969; Wolff, 1963, 1966) and never or very rarely while the infant was fully awake or deeply asleep (quiet sleep). For these reasons, REM endogenous smiling is considered one of the most well-circumscribed state-related behaviors found in the neonatal period (Emde \& Harmon, 1972; Emde \& Koenig, 1969b).

A recent article by Messinger et al. (2002) enlarged our knowledge and raised new questions about the form of neonatal smiling. This observational study, using an anatomically based facial coding system (Baby Facial Action Coding System [FACS]; Oster, 2006), indicated that Duchenne smiling (Ekman, Davidson, \& Friesen, 1990; Ekman \& Friesen, 1982; Frank, Ekman, \& Friesen, 1993), which is the co-occurrence of the contraction of the facial muscles zygomaticus major (lip corner raising, AU 12) and orbicularis oculi (cheek raising, AU 6), is present in sleeping neonates in more or less the same proportions as simple (non-Duchenne) smiling, which is the contraction of zygomaticus major alone (lip corner raising, AU 12).

These findings suggest that smiling has a different developmental trajectory than has been recognized heretofore. Some studies have previously reported anecdotal observations of the Duchenne smile in premature (Oster, 2006) and full-term newborns (Emde et al., 1971; Wolff, 1987), but it was generally maintained that this particular smile was very rare in neonates. Smiling at birth was typically described without reference to the action of the circular muscle surrounding the eyes (Korner, 1969) and it has often been suggested that smiling involving this muscle (which today we define as a Duchenne smile) is a more mature form of smiling that 
is not present in neonates and appears at some later developmental point with the emergence of social smiling (Oster \& Ekman, 1978; Sroufe, 1996; Wolff, 1987). More recently, a dynamic system perspective on the development of facial expressions (Camras, 1992; Fogel \& Thelen, 1987) reinforced this presumed early developmental trajectory of smiling, suggesting a progressive developmental synergistic relation between the facial muscles zygomaticus major and orbicularis oculi (Messinger, Fogel, \& Dickson, 1997).

The results reported by Messinger and coworkers (2002) differed from those of Emde (Emde \& Koenig, 1969a, 1969b), Korner (1969), and Wolff (1963, 1966, 1987), not only with regard to the presence of Duchenne smiles at birth, but also with regard to other temporal characteristics of neonatal smiling. Emde and Koenig (1969a, 1969b), for example, reported a mean rate of 1.1 neonatal smiles (see Emde \& Harmon, 1972) per 10 min, approximately a quarter of that recorded by Messinger and coworkers (2002). Also mean smile events of 2 to $3 \mathrm{sec}$ reported by Emde, Wolff, and Korner were long with respect to the mean duration, around 1 sec, observed in the Messinger et al. (2002) study.

It is likely that these differences stem from the different techniques used to code smiling in these studies. Early studies were conducted prior to the development of anatomically based coding systems (e.g., MAX [Izard, 1979] and FACS [Ekman \& Friesen, 1978]), prior to the introduction of improved technology (e.g., slow-motion digital video), and prior to contemporary research distinguishing between different types of smiling. Specifically, early researchers identified "smiling" as a facial pattern that typically had a duration of more than 1 sec (see Emde \& Koenig, 1969a, 1969b) and was recognizable in naturalistic situations. Messinger et al. (2002), however, identified "smiles" as the product of zygomaticus major contraction digitally coded frame by frame on the basis of the anatomical and technical criteria contained in the FACS (Ekman \& Friesen, 1978) as applied to infants (Oster, 2006). One possibility is that the different rate per minute and mean duration of smiling in the Messinger et al. and Emde et al. studies indicates that newborns engage in a certain amount of zygomatic activity that is not easily recognized as smiling in a naturalistic situation.

In sum, the differences between recent and early studies suggest that a combination of the methodological procedures adopted in the literature, such as an anatomically based microanalytic coding of smiling (see Messinger et al., 2002), associated with a real-time smile recognition task (see, e.g., Emde \& Koenig, 1969a, 1969b) might offer new insights concerning the origins and the characteristics of the emerging facial pattern of smiling.

To further explore the nature of early smiling, we conducted two studies analyzing neonates' anatomically coded zygomatic muscle activity (Study 1 ) and smiling behavior as recognized by naive observers (Study 2). The facial behavior of newborn infants was videorecorded when they were in four different behavioral states (alertness, quiet sleep, active sleep, and drowsiness) with the aim of exploring 
whether the zygomatic activity, as coded by a microanalytic and anatomically based facial coding system (Study 1) as well as recognized as smiling by naive observers (Study 2), is a prototypical neonatal state-dependent phenomenon, as maintained in the literature (see Emde \& Harmon, 1972; Emde \& Koenig, 1969a, 1969b; Korner, 1969; Wolff, 1963, 1966).

In the first study, the distributions of the facial actions that determine Duchenne and simple or non-Duchenne smiles, lip corner raising (AU 12) and lip corner raising with cheek raising (AU $6+\mathrm{AU} 12$ ), respectively, were measured with an anatomically based coding system, the Baby FACS by Oster (2006). We then examined whether the frequency and mean duration of the two facial configurations differ in neonates in different behavioral states.

In the second study, naive observers viewed the same videorecorded material in a computerized smile recognition task. The aim was to identify what types of zygomatic activity, anatomically coded in Study 1, were recognized as smiling. As in Study 1, we examined whether the frequency and the mean duration of recognized smiling differed in the different behavioral states. Finally, contrasts between the data obtained in Study 1 and in Study 2 permitted us to investigate whether the presence of a brief activity of the zygomaticus major, not recognizable as smiling, was produced by the newborn infants we examined.

\section{STUDY 1}

\section{Methods}

\section{Participants}

A group of 32 newborns (14 girls; $M$ age $=25.6 \mathrm{hr}$ postnatal, $S D=15.3$ ) who showed 6 continuous minutes of a stable behavioral state (Brazelton, 1983; Prechtl \& Beintema, 1964; Wolff, 1987) was selected from a large sample of healthy full-term (39-41 weeks gestational age) newborn infants $(N=54)$ videorecorded at the maternity ward of the Pediatric Clinic of the University of Padova.

Eight newborns ( 4 girls; $M$ age $=18.8 \mathrm{hr}, S D=13.8$ ) were in a quiet sleep state (regular sleep or non-REM sleep), 8 newborns ( 3 girls; $M$ age $=23.4 \mathrm{hr}, S D=13.8$ ) were in an active sleep state (irregular, paradoxical, or REM sleep), 8 (4 girls; $M$ age $=21.5 \mathrm{hr}, S D=13.7$ ) were in a state of alertness (quiet waking or alert inactivity), and 8 ( 3 girls; $M$ age $=37.4 \mathrm{hr}, S D=19.8)$ were in a state of drowsiness.

The remaining 22 newborns were eliminated from the sample because they showed an unstable behavioral state during the videorecording $(n=13)$, showed a behavioral state (active waking or alert activity) insufficiently represented to permit a meaningful comparison with the other states considered $(n=3)$, or because of inadequate video quality $(n=6)$. Informed consent was obtained from the infants' parents. 


\section{Procedure}

The newborn infants were videorecorded for $6 \mathrm{~min}$ to $20 \mathrm{~min}$. Videorecordings captured a full-screen image of the neonates' faces as they were sitting in an infant seat. Time accurate to the tenth of a second was inserted onto this image. Videotaping took place during the hour and a half preceding the infants' scheduled feeding times in a quiet, semidarkened room in a secluded part of the newborn nursery.

Behavioral states coding. Behavioral states (quiet sleep, active sleep, alertness, active waking, crying, and drowsiness) were defined in terms of the conjunction of behavioral conditions, incorporating salient features of several well-known coding systems (Brazelton, 1983; Prechtl \& Beintema, 1964; Wolff, 1987). Quiet sleep (regular sleep or non-REM sleep) was characterized by respiration that is regular in rhythm and constant in amplitude, firmly closed eyelids, and no movements except startles or sudden jerks. Active sleep (irregular, paradoxical, or REM sleep) was characterized by intermittent eye movements under closed lids, irregular respiration, and small movements smoother and more controlled than those in quiet sleep. Alertness (alert inactivity or quiet waking) was characterized by minimal motor activity, open eyes, and attention directed toward external stimuli. Active waking (alert activity) was characterized by considerable motor activity involving the limbs, trunk, face, and head, and the eyes were open. Crying was characterized by vigorous vocalizations; the face was contorted into a cry grimace and might be flushed bright red. In drowsiness, the infant was relatively inactive, the eyes occasionally opened and closed intermittently and had a dull, glazed appearance (Brazelton, 1983; Prechtl \& Beintema, 1964; Wolff, 1987).

Two independent coders evaluated behavioral states during the videorecordings at the nursery. Then, infants judged in a stable behavioral state were evaluated again in the videorecordings themselves by two other independent coders. Only those infants who were judged by the four coders as being in the same behavioral state for at least the 6 min sampled for facial coding were considered (100\% agreement among the coders). The four states that occurred sufficiently in the sample to be considered were quiet sleep, active sleep, alertness, and drowsiness.

Facial coding. Frame-by-frame (one frame $=33 \mathrm{msec}$ ) videotape coding was carried out by three reliable Maximally Discriminative Facial Movement Coding System (MAX; Izard, 1979) coders trained in the relevant FACS (Ekman $\&$ Friesen, 1978) and Baby FACS (Oster, 2006) action units (AUs). The FACS is an anatomically based system for identifying the muscular contractions responsible for changes in facial movement (Ekman \& Friesen, 1978). Baby FACS is a version of the FACS applicable to infants and neonates (Oster, 2006).

Three coders independently coded all bilateral lip corner raising (AU 12) and the presence of a cooccurring bilateral cheek raising (AU 6) during the entire se- 
lected video segments ( $6 \mathrm{~min}$ ) for each of the 32 newborns examined. Coders first identified lip corner raising (AU 12, referred to as lip corner pulling in FACS). This action is produced by the zygomaticus major and is the basis of both Duchenne (Ekman et al., 1990; Frank et al., 1993) and simple (Fogel et al., 2000) or non-Duchenne smiles (Messinger et al., 1999, 2001). It raises the lip corners and the infraorbital triangle (making the cheeks more prominent), and deepens the nasolabial furrow between the nose and cheeks. Coders took pains to distinguish lip corner raising (AU 12) from lip corner tightening (dimpling lateral to the lip corners) produced by the buccinator (AU 14) as well as the rare action of the caninus (AU 13; Oster, 2006).

When an instance of lip corner raising was identified, coders ascertained whether there was cooccurring cheek raising (AU 6). A cooccurring instance of lip corner raising and cheek raising was defined as an instance of lip corner raising that, at any point of its duration, cooccurred with cheek raising. Cheek raising is produced by the contraction of the muscle orbiting the eye socket (orbicularis oculi) with fibers that lie around the eye socket (pars lateralis). This action was distinguished from the movement of the inner portion of the same muscle (pars palpebralis) that tightens the eyelids themselves (AU 7 and AU 44).

Tapes were viewed extensively in slow motion on a professional videocassette recorder (VCR) to distinguish the onset and offset of lip corner raising and the cooccurrence of cheek raising. To find the onset time of lip corner raising (AU 12), coders proceeded until the movement peaked or was clearly visible. They then moved backward until the movement stopped and noted the onset time. They confirmed or adjusted the onset time by moving the tape forward and backward across the estimated onset point. Offset time was found in the same manner. Finally, coders ascertained the cooccurrence of a bilateral cheek raising (AU 6). Maximizing the advantages of microanalysis and with the aim of distinguishing very brief and weak actions of muscles, all visible occurrences of AU 12 and AU 6 were considered.

Episodes of lip corner raising identified in the same temporal window of $5 \mathrm{sec}$ by at least two of the three coders were selected for statistical analysis. ${ }^{1}$ Using this window, we then examined agreement in the timing of these smiles among pairs of coders. The percentage of agreement regarding AU 12 coding, identified as agreement within .5 sec around the onset and the offset times, was calculated on $25 \%$ of the participants. It ranged from $81 \%$ to $100 \%(M=90.7, S D=7.9)$. The percentage of agreement regarding AU 6 coding, identified as agreement on its presence or absence within already-identified AU 12 , ranged from $75 \%$ to $100 \%(M=90.1, S D=$ 10.9).

\footnotetext{
${ }^{1}$ We applied the rule that a reliably coded event be coded by at least two independent observers. We used this criterion to avoid overestimating the occurrence of AU 12 by using the decision rule "at least one out of the three coders." In addition, we did not use the decision rule "at least three out of three coders" to avoid underestimating the occurrence of AU 12.
} 
Regarding the estimation of the lip corner raising duration, when there was less than $.5 \mathrm{sec}$ difference between the onset or offset time of the identified AU 12 by the coders, mean onset times or offset times were calculated. When the difference between onset times or between offset times exceeded $.5 \mathrm{sec}$, the coders reached consensus on timing through weekly coding meetings at which each disagreement was discussed. This procedure produced a consensus protocol that was used for statistical analyses. We chose this conservative and time-consuming procedure because it seemed to improve the reliability of the coding and its sensitivity to AU $12 \mathrm{~s}$ of very brief duration.

\section{Data Analysis}

For overall analyses, the frequency (rate per minute), and the mean duration of lip corner raising (AU 12) and the cooccurrence of lip corner raising with cheek raising (AU 6 + AU 12) in each of the behavioral states were obtained. Throughout, if an infant did not show a given facial configuration, the duration of this configuration was treated as missing. We first asked whether zygomatic activity (AU 12 and AU $6+\mathrm{AU}$ 12) was restricted to the states of active sleep and drowsiness and whether the durations of these actions were longer during these states than other states. We then asked whether lip corner raising was more likely to occur in the presence of cheek raising (AU $6+\mathrm{AU}$ 12) than it was to occur alone (AU 12) and whether lip corner raising that occurred with cheek raising (AU $6+\mathrm{AU} 12)$ had a longer duration than lip corner raising alone (AU 12). These last comparisons were carried out between and within behavioral states and then overall. Differences between the states were examined with Mann-Whitney $U$ tests. Friedman tests were adopted to examine matched differences in the occurrence and duration of different types of facial configurations of the same infant within states. In all of these nonparametric analyses, the infant was the unit of analysis.

Regarding duration, we supplemented analyses by infant with analyses using AU 12 as the unit of analysis. These analyses included a contrast of the duration of different types of facial actions (AU 12 vs. AU $6+$ AU 12) in different behavioral states. This comparison was carried out between and within behavioral states and then overall using a nonparametric between-subject analysis, the Mann-Whitney $U$ tests. In these analyses, the total number of episodes of lip corner raising (AU 12 ) and lip corner raising with cheek raising (AU $6+$ AU 12) constituted the sample size.

\section{Results}

Table 1 presents the number and percentage of newborns who showed lip corner raising (AU 12) and lip corner raising with cheek raising (AU $6+\mathrm{AU} 12$ ), and the frequency and mean duration of these actions in the four behavioral states exam- 
TABLE 1

Zygomaticus Major Activity Coded in the Different Behavioral States

\begin{tabular}{|c|c|c|c|c|c|c|c|c|c|c|c|c|c|c|}
\hline \multirow{3}{*}{$\begin{array}{l}\text { Behavioral } \\
\text { States }\end{array}$} & \multicolumn{7}{|c|}{$\begin{array}{l}\text { Lip Corner Raising } \\
(A U 12)\end{array}$} & \multicolumn{7}{|c|}{$\begin{array}{c}\text { Lip Corner Raising With Cheek Raising } \\
(A U 6+A U 12)\end{array}$} \\
\hline & \multicolumn{2}{|c|}{ Infants } & \multirow{2}{*}{$\begin{array}{l}\text { No. of } \\
\text { Events }\end{array}$} & \multicolumn{2}{|c|}{$\begin{array}{l}\text { Rate Per } \\
\text { Minute }\end{array}$} & \multicolumn{2}{|c|}{ Duration } & \multicolumn{2}{|c|}{ Infants } & \multirow{2}{*}{$\begin{array}{l}\text { No. of } \\
\text { Events }\end{array}$} & \multicolumn{2}{|c|}{$\begin{array}{l}\text { Rate Per } \\
\text { Minute }\end{array}$} & \multicolumn{2}{|c|}{ Duration } \\
\hline & No. & $\%$ & & $M$ & $S D$ & $M$ & $S D$ & No. & $\%$ & & $M$ & $S D$ & $M$ & $S D$ \\
\hline Alertness & $6 / 8$ & $75 \%$ & 11 & .23 & .22 & 1.17 & .65 & $7 / 8$ & $88 \%$ & 14 & .29 & .23 & 1.64 & .63 \\
\hline Drowsiness & $8 / 8$ & $100 \%$ & 29 & .58 & .43 & 1.36 & .72 & $5 / 8$ & $63 \%$ & 18 & .40 & .44 & 1.89 & .51 \\
\hline Active sleep & $8 / 8$ & $100 \%$ & 32 & .69 & .54 & 1.51 & .50 & $8 / 8$ & $100 \%$ & 25 & .50 & .18 & 2.25 & .48 \\
\hline Quiet sleep & $4 / 8$ & $50 \%$ & 10 & .21 & .26 & 1.46 & .62 & $4 / 8$ & $50 \%$ & 4 & .08 & .09 & 1.44 & .45 \\
\hline
\end{tabular}

Note. $\quad N=32$. The number of infants refers to the number who produced the facial action. Number of events refers to the total number of AU 12 and AU 6 + AU 12 coded. Mean durations are expressed in seconds. Statistical comparisons are found in the text.

ined. The total number of events $(N=143)$ coded in the different behavioral states is also reported in Table 1. Zygomaticus major activity was observed in all behavioral states. Specifically, lip corner raising, with or without cheek raising, was seen in at least half of the infants in each behavioral state.

Lip corner raising (AU 12): Comparisons between behavioral states. Newborns in active sleep produced more frequent lip corner raising alone than did alert newborns $(U=10.5, p=.022)$ and newborns in quiet sleep $(U=52.5, p=$ .029). Newborns in the drowsy state also produced lip corner raising alone more frequently than newborns in an alert state $(U=12.5, p=.036)$ and than newborns in quiet sleep $(U=14, p=.054)$, although this last comparison was on the borderline of statistical significance. No differences were found in the mean durations of the lip corner raising alone that occurred in the different behavioral states using these nonparametric techniques that utilized the infant as the unit of analysis. This absence of significant results in duration was corroborated with the nonparametric analyses in which AU 12 was used as the unit of analysis.

Lip corner raising with cheek raising ( $A \cup 6+A U$ 12): Comparisons between behavioral states. We found that lip corner raising with cheek raising occurred more frequently during active sleep than quiet sleep $(U=64, p=.001)$. In addition, there was a trend for newborns in active sleep to more frequently produce the lip corner raising and cheek raising (AU $6+\mathrm{AU}$ 12) facial configuration than newborns in the alert state, but this contrast did not reach statistical significance ( $U$ $=15.5, p=.075)$. With respect to mean duration, contrasts between the states revealed that neonates in active sleep showed lip corner raising associated with 
cheek raising (AU $6+$ AU 12) for longer periods of time than did newborns in quiet sleep $(U=31, p=.011)$ and alert newborns $(U=11, p=.049)$. Supplementary analyses conducted using AU 12 as the unit of analysis confirmed that lip corner raising with cheek raising were of longer duration during active sleep than during alertness $(U=97, p=.022)$, but did not confirm that the active sleep AU $6+\mathrm{AU}$ $12 \mathrm{~s}$ were of longer duration than the quiet sleep AU $6+\mathrm{AU} 12 \mathrm{~s}(U=71, p=.184)$.

Lip corner raising $(A \cup 12)$ versus lip corner raising with cheek raising
$(A \cup 6+A U$ 12): Comparisons within behavioral states and overall. With respect to contrasts of frequency within each of the behavioral states, no significant differences were found regarding the two facial configurations. Comparisons of duration revealed that the lip corner raising and cheek raising association had a longer mean duration than lip corner raising alone (AU 12) during active sleep ( $F r$ $=8, p=.005$ ) but not during the other states. The same significant result was obtained in the between-subject analysis that adopted the AU 12 as the unit $(U=254$, $p=.019$ ).

We then examined contrasts between lip corner raising alone (AU 12) and lip corner raising with cheek raising (AU $6+$ AU 12) over all behavioral states. Eighty-one percent of the neonates showed lip corner raising alone, whereas 75\% showed lip corner raising with cheek raising. No differences were found between the frequency of lip corner raising $(M=.43, S D=.42)$ and lip corner raising with cheek raising $(M=.32, S D=.30)$. The Friedman test revealed that lip corner raising with cheek raising had a greater mean duration $(M=1.86 \mathrm{sec}, S D=.59)$ than lip corner raising alone ( $M=1.38 \mathrm{sec}, S D=.60 ; n=21, d f=20, F r=8.05, p=$ $.005)$. This difference in duration over all behavioral states was confirmed using a nonparametric between-subject analysis that treated AU 12 and AU $6+$ AU 12 as the unit of analysis $(n=143, d f=1, U=1,983, p=.034)$.

\section{Discussion}

There were three main results of Study 1. First, the presence at birth of the association between lip corner raising and cheek raising (Duchenne smile) was confirmed, corroborating the findings of Messinger and coworkers (2002).

Second, zygomatic activity did not occur only during active sleep and drowsiness, as expected, but also occurred during alertness and quiet sleep. However, these two facial configurations were exhibited more frequently during active sleep and drowsiness rather than during the other states considered. These data do not support the hypothesis that the zygomaticus major activity, the essential facial muscular action necessary to produce a smile, is a state-related behavior, as maintained in the literature (Emde \& Koenig, 1969a, 1969b; Korner, 1969; Wolff, 1963, 1966). 
Third, the analysis also revealed that lip corner raising with cheek raising (AU 6 + AU 12) was of longer duration than lip corner raising alone. This effect was seen when summing over all behavioral states, as well as when the neonates were in active sleep, but not in the other states considered. In addition, lip corner raising with cheek raising produced during active sleep was of longer duration than the same facial pattern in almost all the other behavioral states. During active sleep, the cooccurrence between the two facial actions was more enduring. These data suggest a possible association between active sleep and lip corner raising with cheek raising.

To further investigate the characteristics of neonatal smiling, a second study was conducted. The main aim was to identify what types of zygomatic activity (lip corner raising and lip corner raising with cheek raising), anatomically coded in Study 1, would be recognized as smiling by naive observers.

\section{STUDY 2}

In the second study, naive observers were presented with the same 32 video segments of 6 min that had been coded in Study 1. The naive observers were asked, while viewing the videorecordings at normal speed through a computer interface, to indicate when the infants were smiling. The type of smile recognized-that is, a Duchenne smile (specified by lip corner raising with cheek raising, AU $6+$ AU 12) or a simple or non-Duchenne smile (specified by lip corner raising alone, AU 12) - as well as the duration of the smile were obtained from the coding protocol of Study 1 . That is, we categorized each smile identified by the naive observers as a recognized Duchenne or non-Duchenne smile, and tabulated its previously coded duration. This redescription of the data permitted us to perform the same statistical analyses carried out in Study 1, but this time with respect to recognized smiles.

The first aim of Study 2 was to examine the degree to which the infant's behavioral state influenced the form and the rate of neonatal smiles recognizable by naive observers. It is possible that the state-related distributions of smiling reported in the literature (Emde \& Harmon, 1972; Emde \& Koenig, 1969a, 1969b; Korner, 1969), although not confirmed by coding in Study 1, characterize instead only the zygomatic activity recognized as smiling.

The second aim of the study was to further explore the relation, which emerged in Study 1, between lip corner raising with cheek raising (AU $6+$ AU 12) and active sleep. We wished to determine whether recognized Duchenne smiling tended to occur more frequently during active sleep than in the other states considered, and whether it had a longer mean duration in this state than simple or non-Duchenne smiling.

The third and most important aim was to investigate the presence of zygomatic contractions not recognized as smiling. Contrasts between the data obtained in 
Study 1 and in Study 2 permitted us to test the degree to which anatomically coded zygomatic activity was recognized as smiling by naive observers. This contrast indicates whether zygomatic activity produced by neonates is limited to facial patterns recognizable as smiling or whether zygomaticus major contractions, not perceived as meaningful expressions and possibly of a brief duration, are present in very early life.

\section{Method}

\section{Participants}

Participants were 48 volunteer undergraduate university students (29 women, $M$ age $=24$ years). They had no knowledge of the issue under investigation. Each of them was tested individually in a quiet and semidarkened room in a university laboratory.

\section{Stimuli}

The same 32 six-min videorecorded segments analyzed in Study 1 were used as stimuli.

\section{Apparatus}

Participants' judgments were recorded through an apparatus composed of a VCR and a 14-in. monitor remotely controlled by a personal computer through a serial driver. Software written for this apparatus reproduced a virtual copy of the front panel of the VCR with which the observer interacted during the trial. The apparatus permitted a bidirectional exchange of data and commands (coded as ASCII strings) between the VCR and the personal computer. The timing of the computer was synchronized with the timing of each of the 32 six-min video segments with the aim of easily linking each recognized smile to the correspondent lip corner raising (with or without cheek raising) coded in Study 1. The software that implemented the experimental protocol was realized through a graphic programming tool (Labview, National Instruments).

\section{Procedure}

Each observer was instructed to place the cursor of the mouse on a big red virtual button, which appeared on the computer screen, to maintain the cursor in that position during the entire trial, and to look steadily at the VCR monitor. The observer was then instructed to click the computer mouse as soon as possible after the appearance of each smile of the baby. After a brief familiarization with the apparatus, the experimenter invited the observer to start the trial clicking on the Play button located on the virtual panel. The program recorded all the timing correspond- 
ing to the key presses on the mouse by the observer. Each of 48 naive coders viewed two 6-min video segments; each of those segments showed a neonate in a different behavioral state. Each of the 32 segments was presented to three observers, so the total number of segments seen by the observers was 96 .

Smile coding. Timing regarding recognized smiles (observer's key presses) was linked to the lip corner raising (AU 12) and lip corner raising and cheek raising (AU $6+$ AU 12) coded in Study 1. This permitted us to examine the duration and the type of zygomatic activity (with or without cheek raising) that had been recognized by naive observers as smiles. Specifically, each lip corner raising alone, as coded in Study 1, recognized by the naive observers as a smile was considered a recognized simple or non-Duchenne smile. Each lip corner raising with cheek raising recognized as a smile was considered a recognized Duchenne smile. Following the decision rule adopted in Study 1, we utilized only smiles recognized by at least two out of the three naive observers for statistical analyses (see also footnote 1).

\section{Data Analysis}

The analyses we carried out were the same as those conducted in Study 1, specifically contrasts of mean frequency (rate per minute) and mean duration (measured in milliseconds). We first asked whether smiling was recognized only when the neonates were in a state of active sleep or were drowsy. We then asked whether lip corner raising (simple smile) or lip corner raising with cheek raising (Duchenne smile) was more likely to be recognized as smiling. We also asked whether recognized smiles that had been coded as lip corner raising with cheek raising (Duchenne smile) lasted longer than recognized smiles that had been coded as lip corner raising alone (simple smile). These comparisons were carried out between and within the behavioral states and then overall. Comparisons between the behavioral states were examined by nonparametric Mann-Whitney $U$ tests, whereas withinsubjects variable contrasts were run by nonparametric Friedman tests. If an infant did not show a recognized facial configuration, the duration of this configuration was treated as missing.

As in Study 1, we supplemented analyses of duration by infants with analyses using smile as the unit of analysis. These included a contrast of the duration of different types of smiles in different behavioral states. These last comparisons were carried out between and within behavioral states and then overall using a nonparametric between-subject analysis, the Mann-Whitney $U$ tests. In these analyses the total number of recognized simple and Duchenne smiles constituted the sample size.

In addition, to investigate the differences between expert facial coders and naive observers in identifying zygomatic activity and recognizing smiling behavior in 
the same data set, we contrasted the rate per minute of lip corner raising (AU 12) and lip corner raising with cheek raising (AU $6+$ AU 12) as coded in Study 1 with the same behavioral measures regarding the smiles (simple and Duchenne smiles) as recognized in Study 2. These contrasts were conducted using a Friedman test. Finally, to address whether duration was critical in the process of smile recognition, we contrasted recognized versus unrecognized simple and Duchenne smiles. These last contrasts were conducted, over all and within behavioral states, using the nonparametric Mann-Whitney $U$ test.

\section{Results and Discussion}

Table 2 refers to the number and percentage of neonates that were identified by at least two out of three naive observers as showing the smile and to the number, rate per minute, and mean duration of the recognized smiles in the four behavioral states. As shown in Table 2, naive observers identified smiling in all the behavioral states examined.

\section{Recognized Smiles: Comparisons Between and Within Behavioral States}

Recognized simple smiles: Comparisons between behavioral states. Simple or non-Duchenne smiles were recognized more often in active sleep than quiet sleep ( $U=48, p=.046)$. No other significant differences regarding the frequency (rate per minute) were found. There were also no differences in the mean duration of recognized simple smiles in different states, whether using the infant or the smile as the unit of analysis.

Recognized Duchenne smiles: Comparisons between behavioral states. Duchenne smiles were recognized more often in active sleep than quiet sleep $(U=$ $61, p=.001)$. In addition, newborn infants in active sleep more frequently produced a recognized Duchenne smile than did alert newborns $(U=6, p=.004)$ and drowsy newborns $(U=48.5, p=.074)$, although this effect was on the borderline of statistical significance. Regarding mean duration, statistical contrasts revealed that newborns in active sleep produced recognized Duchenne smiles for a longer duration than did alert newborns $(U=0, p=.037)$ and, on the borderline of statistical significance, than did newborns in the quiet sleep state $(U=15, p=.068)$. Supplementary analyses conducted using the smile as the unit of analysis confirmed that recognized Duchenne smiles were of longer duration during active sleep than during alertness $(U=4.5, p=.033)$. 


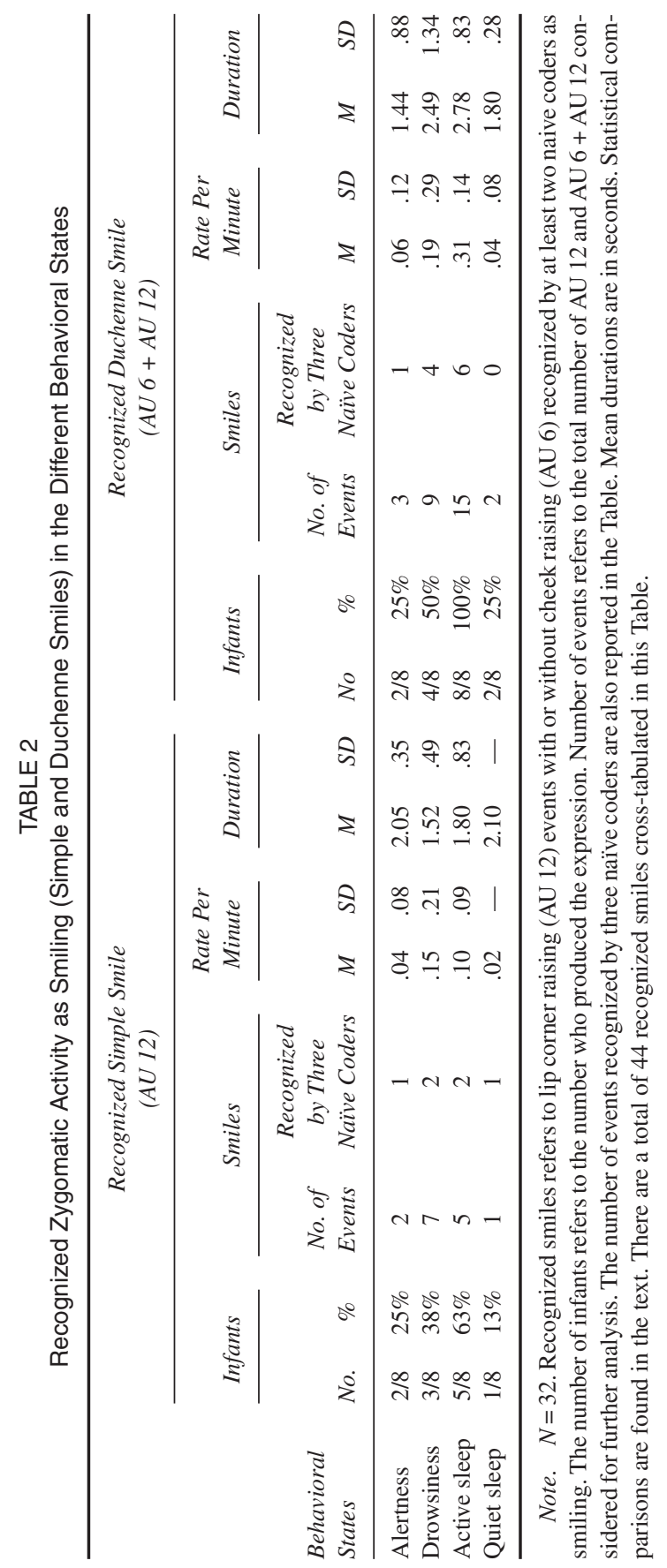


Recognized simple smiles versus recognized Duchenne smiles: Comparisons within behavioral states and overall. With respect to contrasts regarding frequency (rate per minute), statistical analyses revealed that Duchenne smiles were recognized more frequently than simple smiles when the neonates were in a state of active sleep $(F r=4.5, p=.034)$. No other differences were found within each state regarding the frequency. The same was true for comparisons of mean duration, either using the infant or the smile as the unit of analysis.

Then, we examined contrasts between recognized simple smiles and recognized Duchenne smiles over all behavioral states. Thirty-four percent of the newborns produced recognized simple smiles, whereas 50\% showed recognized Duchenne smiles. With respect to the frequency measure, no differences were found on rate per minute between simple smiles $(M=.08, S D=.13)$ and Duchenne smiles $(M=.15, S D=.20)$. This was also the case for the comparison of mean duration, in which recognized Duchenne smiles $(M=2.42 \mathrm{sec}, S D=.97)$ and recognized simple smiles $(M=1.79 \mathrm{sec}, S D=.62)$ did not differ. These results, in which the infant was the unit of analysis, were unchanged when the smile was treated as the unit of analysis.

With respect to the first aim of Study 2, these data do not support the hypothesis that recognized neonatal smiling occurs exclusively during active sleep and drowsiness. Although smiling was recognized more frequently during these states, neonates in both alertness and quiet sleep states produced recognized simple and Duchenne smiles. With respect to the second aim of Study 2, recognized Duchenne smiles during active sleep were more frequent than in the other states considered and tended also to have a longer mean duration, suggesting an association between active sleep and a frequent and relatively long-lasting Duchenne smile configuration.

\section{Comparison Between the Anatomical Facial Coding (Study 1) and the Recognition Study (Study 2)}

Lip corner raising (AU 12) versus recognized simple smile in the different behavioral states. With respect to the frequency measure (rate per minute), the analyses revealed a significant difference between the lip corner raising alone (AU 12) anatomically coded and the recognized simple smiles when the neonates were in an active sleep state $(F r=8, p=.005)$, as well as when the neonates were drowsy $(F r=6.12, p=.013)$. In particular, expert coders more frequently coded lip corner raising (AU 12) than naive observers recognized simple smiles, either when the neonates were in active sleep or when they were drowsy. A similar trend was observed with respect to the alertness group $(F r=3.13, p=.077)$.

Table 3 refers to the number and the mean duration of unrecognized simple and Duchenne smiles in the four behavioral states. No differences were found between the mean durations of recognized (see Table 2) and unrecognized simple smiles. 
TABLE 3

Unrecognized Zygomatic Activity as Smiling (Simple and Duchenne Smiles) in the Different Behavioral States

\begin{tabular}{|c|c|c|c|c|c|c|c|c|}
\hline \multirow[b]{3}{*}{$\begin{array}{l}\text { Behavioral } \\
\text { States }\end{array}$} & \multicolumn{4}{|c|}{$\begin{array}{l}\text { Unrecognized } A U 12 \\
\text { as Simple Smile }\end{array}$} & \multicolumn{4}{|c|}{$\begin{array}{c}\text { Unrecognized } A U 6+A U 12 \\
\text { as DuchenneSmiles }\end{array}$} \\
\hline & \multicolumn{2}{|r|}{ Smiles } & \multicolumn{2}{|c|}{ Duration } & \multicolumn{2}{|r|}{ Smiles } & \multicolumn{2}{|c|}{ Duration } \\
\hline & $\begin{array}{l}\text { No. of } \\
\text { Events }\end{array}$ & $\begin{array}{l}\text { Not Recognized } \\
\text { by Any of the } \\
\text { Three Observers }\end{array}$ & $M$ & $S D$ & $\begin{array}{l}\text { No. of } \\
\text { Events }\end{array}$ & $\begin{array}{l}\text { Not Recognized } \\
\text { by Any of the } \\
\text { Naïve Coders }\end{array}$ & $M$ & $S D$ \\
\hline Alertness & 9 & 3 & 1.18 & .61 & 11 & 4 & 1.37 & .70 \\
\hline Drowsiness & 22 & 11 & 1.49 & 1.09 & 9 & 2 & 1.16 & .59 \\
\hline Active sleep & 27 & 12 & 1.44 & .75 & 10 & 6 & 1.36 & .71 \\
\hline Quiet sleep & 9 & 6 & 1.32 & .67 & 2 & 2 & 1.13 & .46 \\
\hline
\end{tabular}

Note. Unrecognized smiles refers to lip corner raising events with or without cheek raising not recognized as smiling ( 0 hits) or recognized by only one naive coder ( 1 hit). Number of events refers to the total number of AU 12 and AU $6+$ AU 12 considered for further analysis. The number of events not recognized by any of the three naïve coders are also reported in the Table. Mean durations are in seconds. Statistical comparisons are found in the text. There are a total of 99 unrecognized smiles cross-tabulated in this Table.

Lip corner raising with cheek raising ( $A U 6+A U$ 12) versus recognized Duchenne smile in the different behavioral states. Lip corner raising and cheek raising (AU $6+$ AU 12) was coded by expert coders at a higher rate per minute than the same facial configuration was recognized by naive observers as a smile. This difference was evident when the neonates were alert $(F r=4.5, p=$ $.034)$, in active sleep $(F r=4.5, p=.034)$, and, at a trend level, when neonates were in a drowsy state $(F r=3.1, p=.077)$. Significant differences were also found in the durations of these smiles (see Table 3$)$. During active sleep $(U=16, p=.001)$ and during drowsiness $(U=12, p=.012)$, recognized Duchenne smiles were of longer duration than unrecognized Duchenne smiles.

Over all behavioral states. Independent of behavioral state, the frequency of lip corner raising (AU12) identified by the expert coders $(M=.43)$ was greater than the frequency of simple smile recognized by the naive observers $(M=.08, F r$ $=18, p=.0001)$. In addition, the expert coders more frequently identified lip corner raising with cheek raising (AU $6+\mathrm{AU} 12)$ configurations $(M=.32)$ than these same configurations were recognized as Duchenne smiles by naive observers $(M=$ $.15, F r=11.28, p=.001)$. Also the durations of recognized and unrecognized smiles were significanty different regarding both simple $(U=317, p=.026)$ and Duchenne smiles $(U=154, p=.0001)$. In particular, recognized smiles were of longer duration than unrecognized smiles for both simple (1.76 vs. 1.40) and Duchenne smiles (2.40 vs. 1.30). 
With respect to the third aim of Study 2, we found that only a subset of the zygomatic activity (lip corner raising with or without cheek raising) identified by expert coders were recognized as smiling (Duchenne and non-Duchenne smiles) by naive observers. This means that newborns engaged in some zygomatic activity that was not recognized as smiling. Neither the presence or absence of the Duchenne marker, nor the newborn's behavioral state distinguished activity of the zygomaticus major that was and was not recognized as smiling. Instead, longer duration contributed to smile recognition.

\section{GENERAL DISCUSSION}

Combining microanalytic anatomical coding of zygomaticus major activity (Study 1) with a smile recognition study (Study 2) offered new insights into the nature of neonatal smiling. Three main results were obtained. First, we demonstrated the presence of a brief zygomaticus major activity (with or without cheek raising) distinguished by expert coders but not recognized as smiling by the naive observers. Second, we found that coded lip corner raising (with or without cheek raising) as well as recognized smiling (Duchenne and non-Duchenne) occurred in all behavioral states examined — not only in active sleep—and so did not appear to be a prototypical state-related behavior. Third, we found that lip corner raising with cheek raising (Study 1) as well as the recognized Duchenne smiling (Study 2) tended to occur more frequently and to have a longer mean duration during active sleep (irregular, paradoxical, or REM sleep) than in the other states considered.

With respect to the first main result, the presence of zygomatic activity not recognized as smiling emerged by contrasting data obtained in Study 1 with data obtained in Study 2. Coded lip corner raising with or without cheek raising not recognized as smiling was typically briefer than the zygomatic activity recognized as smiling. This may explain why early investigations documented less frequent, longer lasting smiles (Emde \& Koenig, 1969a, 1969b; Korner, 1969; Wolff, 1963, 1966) than more recent investigations (Messinger et al., 2002).

The presence of brief zygomatic contractions characterized all behavioral states. In fact, zygomatic activity not recognized as smiling was more frequent when overall zygomatic contractions were more frequent, so that it peaked during active sleep and drowsiness, and was at lower levels in alertness and quiet sleep. This suggests that contractions of the zygomaticus major that were not recognized as smiling pertained to the same processes of recognized smiling and had similar distributions in the different behavioral states. This brief activity does not appear dissimilar to the phenomenon of microexpressions (Ekman, 2003), which are brief (sometimes visible only in one movie frame), involuntary, and incomplete facial emotional expressions that occur on adults' faces. However, contrary to what has been reported in adult studies, brief neonatal zygomatic activity appears to be very 
frequent. A limitation of this study is that the muscular intensity of AU 6 and AU 12 was not coded. Coding of the muscular intensity of both lip corner raising and cheek raising might clarify whether these brief periods of zygomatic activity were also of a low muscular intensity.

Regarding the second main result, we found that the zygomatic activity (Study 1) as well as the recognized smiles (Study 2) did not only occur in active sleep and drowsiness as has been reported (Emde \& Koenig, 1969a, 1969b; Korner, 1969; Wolff, 1963, 1966). These data, in particular, are not consistent with the early literature that reports that in the first postnatal week the infant does not smile during waking state (Korner, 1969; Wolff, 1966) or, at least, that smiling is very rarely observed in this state (Wolff, 1987).

How we can explain such differences between early and recent studies? It is possible, as was recognized by Emde and Koenig (1969b), that earlier studies focused primarily on the behavioral state of active sleep, observing the newborn mainly after he or she was fed, and concentrating attention on the relationship between REM states and smiling. This may have led researchers to neglect the state of alertness, which is more difficult to observe at birth and is typically of brief duration (Brazelton, 1983; Prechtl \& Beintema, 1964; Wolff, 1987). In addition, the zygomatic activity, as we have demonstrated, is generally of longer duration during active sleep and drowsiness than during alertness, rendering it more recognizable as smiling in a naturalistic situation.

Regarding the third main result, both the studies revealed a slight but unambiguous relation between active sleep and the lip corner raising with cheek raising configuration (AU $6+$ AU 12), as coded in Study 1, and the Duchenne smile, as recognized in Study 2. In general, both overall AU 6 + AU 12 and recognized Duchenne smiles were more frequent and had a longer mean duration during active sleep than during the other behavioral states. In addition, it was only during active sleep that lip corner raising with cheek raising and recognized Duchenne smiling had longer mean durations, respectively, than lip corner raising alone and than recognized non-Duchenne smiles.

This association is interesting in light of the intimate relationship between active or REM sleep and limbic activation (Maquet et al., 1996; Roffwarg, Muzio, \& Dement, 1966), which is involved in emotional responding. It suggests potential ties between the Duchenne smiles of neonates and the Duchenne smiles of adults (Ekman et al., 1990; Ekman \& Friesen, 1982; Frank et al., 1993) and infants (Fogel et al., 2000; Fox \& Davidson, 1988; Messinger et al., 1999, 2001), which are indexes of positive emotion.

REM sleep is associated in adults with intense neuronal activity, ocular saccades, muscular atonia, and dreaming (Hobson, 1988; Jones, 1991). Maquet et al. (1996) showed that regional cerebral blood flow is positively correlated with REM sleep in pontine tegmentum, left thalamus, both amygdaloid complexes, anterior cingulate cortex, and right parietal operculum. At birth, as reported by Roffwarg 
and collaborators (1966), the amount and variability of autonomic activity observable during active sleep (penile erections, irregular respirations, marked variations in heart rate) is highly suggestive of limbic activation. The role of active sleep (irregular, paradoxical, or REM sleep) at birth is still unclear but the great prominence of this state during early life probably serves to assist the process of central nervous system maturation and differentiation (Marks, Shaffery, Oksenberg, Speciale, \& Roffwarg, 1995; Mirmiran, 1995).

Given this evidence, it is possible to suppose that neonatal Duchenne smiling (lip corner raising with cheek raising) exhibited during active sleep, more than those exhibited in other behavioral states and more than simple or non-Duchenne smiling (lip corner raising alone), may be influenced by limbic system discharges. It is also possible that Duchenne smiles that were particularly stable and enduring may represent the most plausible candidates for being expressions of this limbic activation. As a working hypothesis, these episodes may be conceptualized as brief and unstable expressions of the emerging coordination between a facial motor synergy (the association between the zygomaticus major and the orbicularis oculi activity) and the neural structures that later in development (at about 2 months of age) support an alert and social expression of smiling.

In sum, the frequent motor activity of the zygomaticus major muscles with or without cheek raising and across different behavioral states, seems to appropriately describe the developmental status at birth of the facial pattern that specifies smiling. Sometimes, this early motor activity is very brief; sometimes it is sufficiently enduring to be recognized as a meaningful facial expression. For these reasons, it is likely that neonatal smiling is still a facial pattern in search of a locus of control (neural, cognitive, social, emotional), free to enter into a number of different low-dimensional ensembles following a process of spontaneous pattern formation or a genetic and prespecified pathway.

\section{ACKNOWLEDGMENTS}

We wish to thank the infants and parents who participated in this research, Franco Zacchello and the nursing staff at the Pediatric Clinic of the University of Padua for their invaluable cooperation, and Pietro Scatturin, who developed the apparatus employed in the second study.

\section{REFERENCES}

Ambrose, J. A. (1961). The development of the smiling response in early infancy. In B. M. Foss (Ed.), Determinants of infant behaviour (pp. 179-196). Oxford, UK: Wiley. 
Brazelton, T. B. (1983). Clinics in developmental medicine, No. 88: Neonatal Behavioral Assessment Scale (NBAS) (2nd ed.). Philadelphia: Lippincott.

Camras, L. A. (1992). Expressive development and basic emotions. Cognition and Emotion, 6, 269-283.

Ekman, P. (2003). Emotions revealed: Recognizing faces and feelings to improve communication and emotional life. New York: Times Books.

Ekman, P., Davidson, R. J., \& Friesen, W. V. (1990). The Duchenne smile: Emotional expression and brain physiology II. Journal of Personality and Social Psychology, 58, 342-353.

Ekman, P., \& Friesen, W. V. (1978). Facial Action Coding System (FACS). Palo Alto, CA: Consulting Psychologists Press.

Ekman, P., \& Friesen, W. V. (1982). Felt, false, and miserable smiles. Journal of Nonverbal Behavior, 6, 238-252.

Emde, R. N., \& Harmon, R. J. (1972). Endogenous and exogenous smiling systems in early infancy. Journal of the American Academy of Child Psychiatry, 22, 178-200.

Emde, R. N., \& Koenig, K. L. (1969a). Neonatal smiling and rapid eye movement states. Journal of the American Academy of Child Psychiatry, 8, 57-67.

Emde, R. N., \& Koenig, K. L. (1969b). Neonatal smiling, frowning, and rapid eye movement states: II. Sleep-cycle study. Journal of the American Academy of Child Psychiatry, 8, 637-656.

Emde, R. N., McCartney, R. D., \& Harmon, R. J. (1971). Neonatal smiling in REM states: IV. Premature study. Child Development, 42, 1657-1661.

Fogel, A., Nelson-Goens, G. C., Hsu, H. C., \& Shapiro, A. F. (2000). Do different infant smiles reflect different positive emotions? Social Development, 9, 497-520.

Fogel, A., \& Thelen, E. (1987). Development of early expressive and communicative action: Reinterpreting the evidence from a dynamic systems perspective. Developmental Psychology, 23, 747-761.

Fox, N., \& Davidson, R. J. (1988). Patterns of brain electrical activity during facial signs of emotion in 10-month-old infants. Developmental Psychology, 24, 230-236.

Frank, M. G., Ekman, P., \& Friesen, W. V. (1993). Behavioral markers and the recognizability of the smile of enjoyment. Journal of Personality and Social Psychology, 64, 83-93.

Gewirtz, J. L. (1965). The course of infant smiling in four child-rearing environments in Israel. In B. M. Foss (Ed.), Determinants of infant behavior (Vol. 3, pp. 205-248). New York: Wiley.

Hobson, J. A. (1988). The dreaming brain. London: Penguin.

Izard, C. E. (1979). The maximally discriminative facial movement coding system (MAX). Newark: University of Delaware, Instructional Resources Center.

Jones, B. E. (1991). Paradoxical sleep and its chemical/structural substrates in the brain. Neuroscience, 40, 637-656.

Korner, A. F. (1969). Neonatal startles, smiles, erections, and reflex sucks as related to state, sex, and individuality. Child Development, 40, 1039-1053.

Maquet, P., Péters, J. M., Aerts, J., Delfiore, G., Degueldre, C., Luxen, A., et al. (1996). Functional neuroanatomy of human rapid-eye-movement sleep and dreaming. Nature, 383(6596), 163-166.

Marks, G. A., Shaffery, J. P., Oksenberg, A., Speciale, S. G., \& Roffwarg, H. P. (1995). A functional role for REM sleep in brain maturation. Behavioural Brain Research, 69(1-2), 1-11.

Messinger, D. S., Dondi, M., Nelson-Goens, C. G., Beghi, A., Fogel, A., \& Simion, F. (2002). How sleeping neonates smile. Developmental Science, 5, 49-55.

Messinger, D. S., Fogel, A., \& Dickson, K. L. (1997). A dynamic system approach to infant facial action. In J. A. Russell \& M. Fernandez-Dols (Eds.), The psychology of facial expression (pp. 205-226). New York: Cambridge University Press.

Messinger, D. S., Fogel, A., \& Dickson, K. L. (1999). What's in a smile? Developmental Psychology, 35, 701-708.

Messinger, D. S., Fogel, A., \& Dickson, K. L. (2001). All smiles are positive, but some smiles are more positive than others. Developmental Psychology, 37(5), 642-653. 
Mirmiran, M. (1995). The function of fetal/neonatal rapid eye movement sleep. Behavioural Brain Research, 69(1-2), 13-22.

Oster, H. (2006). Baby FACS: Facial Action Coding System for infants and young children. Unpublished monograph and coding manual. New York University.

Oster, H., \& Ekman, P. (1978). Facial behavior in child development. Minnesota Symposia on Child Psychology, 11, 231-276.

Prechtl, H. F. R., \& Beintema, D. (1964). The neurological examination of the full term newborn. London: SIMP Heinemann.

Roffwarg, H. P., Muzio, J. N., \& Dement, W. C. (1966). Ontogenetic development of the human sleep-dream cycle. Science, 152, 604-619.

Sroufe, A. (1979). Socioemotional development. In J. D. Osofsky (Ed.), Handbook of infant development (pp. 462-516). New York: Wiley.

Sroufe, A. (1996). Emotional development. New York: Cambridge University Press.

Sroufe, L., \& Waters, E. (1976). The ontogenesis of smiling and laughter: A perspective on the organization of development in infancy. Psychological Review, 83, 1326-1344.

Wolff, P. H. (1963). Observations on early development of smiling. In B. M. Foss (Ed.), Determinants of infant behavior (Vol. 2, pp.113-138). New York: Wiley.

Wolff, P. H. (1966). The causes, controls and organization of behavior in the neonate. Psychological Issues Monograph Series, 5(1). New York: International Universities Press.

Wolff, P. H. (1987). The development of behavioral states and the expression of emotions in early infancy. Chicago: University of Chicago Press. 